\title{
Association between timing and number of antenatal care visits on uptake of intermittent preventive treatment for malaria during pregnancy among Malawian women
}

\author{
Owen Nkoka', Ting-Wu Chuang ${ }^{2}$ and Yi-Hua Chen ${ }^{1 *}$
}

\begin{abstract}
Background: Malaria in pregnancy is a critical public health challenge, and intermittent preventive treatment for malaria during pregnancy (IPTp) has proven to be an effective intervention. However, access to and use of malaria interventions, including IPTp, remains a considerable problem among African women. This cross-sectional study investigated factors, including antenatal care (ANC) attendance (both numbers of visits and timing of the first visit) and socio-demographics, associated with the uptake of the recommended IPTp dose among Malawian women.

Methods: A nationally representative sample of women with a live birth in the 2 years preceding the survey from the Malawi Demographic Health Survey 2015-2016 dataset was analysed. Self-reported data on socio-demographics, ANC attendance and IPTp uptake were collected using a questionnaire and analysed using logistic models.

Results: Of the 6549 included women, 1981 (30.2\%) took the recommended three or more IPTp doses. Despite inadequate ANC visits, early ANC initiation increased the likelihood of these women taking the recommended IPTp dose; women who initiated ANC in the first [adjusted odds ratio $(\mathrm{aOR})=2.24 ; 95 \%$ confidence interval $(\mathrm{Cl})=1.44-3.49$ ] and second $(\mathrm{aOR}=2.19 ; 95 \% \mathrm{Cl}=1.56-3.08$ ) trimesters were more likely to take the recommended IPTp dose compared to late initiators. The effect of the number of ANC visits on IPTp uptake was significant in married women $(\mathrm{aOR}=1.68$, $95 \% \mathrm{Cl}=1.42-1.98)$, and the timing of first ANC visit was associated with IPTp uptake only among rural women $(\mathrm{aOR}=2.13,95 \% \mathrm{Cl}=1.54-2.95)$.

Conclusion: ANC attendance is vital in IPTp uptake. The results highlight the need for health care providers to encourage women, particularly those in high-risk groups, to make frequent ANC visits and receive early ANC initiation to ensure high coverage of the recommended IPTp dose.
\end{abstract}

Keywords: Antenatal attendance, Intermittent preventive treatment, Malaria in pregnancy, Malawi

\section{Background}

Malaria remains a critical public health issue, with particular burden of disease on children aged younger than 5 years and pregnant women [1]. Malaria in pregnancy (MIP) poses a serious threat to mothers, fetuses and newborns, leading to infant and maternal mortality, low

\footnotetext{
*Correspondence: yichen@tmu.edu.tw

${ }^{1}$ School of Public Health, College of Public Health, Taipei Medical University, Taipei, Taiwan

Full list of author information is available at the end of the article
}

birth weight, and maternal anaemia [1]. Globally, MIP is responsible for 10,000 maternal deaths per year [2]. Furthermore, in sub-Saharan Africa, it accounts for 20 and $11 \%$ of stillborn and neonatal deaths, respectively [3, 4].

Malawi is a malaria-endemic country. Between 2009 and 2014, an infection rate of $19.6 \%$ was reported among pregnant women attending their first antenatal care (ANC) service in a peri-urban area of Ndirande, in Blantyre [5]. In addition to the aforementioned impact of MIP, the economic burden of malaria in Malawi is high. An average Malawian family spends approximately US $\$ 17.48$ 
per malaria episode, which is higher than a week's income for most Malawian families [6]. Considering the adverse effects of MIP, the use of intermittent preventive treatment for malaria during pregnancy (IPTp) is one of the most important strategies adopted by the Malawi Ministry of Health (MoH) to reduce the MIP burden [7].

In IPTp, pregnant women are prescribed sulfadoxinepyrimethamine (SP) [8]. The World Health Organization (WHO) recommends that women in malaria-endemic areas should be given the IPTp dose during ANC visits as early as possible in the second trimester and that subsequent doses should be given at 4-week intervals [9]. An appropriate dose of IPTp reduces maternal mortality along with the risks of maternal anaemia and low birth weight [10]. A Malawian study, conducted in Blantyre from July 1997 to April 1999, reported a significant association between SP use and reduced placental malaria parasite prevalence among IPTp non-users (31.9\%) and users $(22.8 \%)$ [11].

In 1993, Malawi became the first country worldwide to implement an IPTp policy, recommending two doses of SP to all pregnant women [12-14], 5 years before the WHO released its IPTp recommendations [15]. Thereafter, the coverage with two doses of IPTp (formerly, the recommended dose) has been relatively high in Malawi compared to the rest of sub-Saharan Africa [16-18]. In $2015,63 \%$ of pregnant women received at least two doses; the percentage of women receiving one IPTp dose increased from $80.7 \%$ in 2006 to $89 \%$ in 2015 [19, 20]. In Malawi, monitoring of the uptake of 3 IPTp doses started in 2014, after IPTp policy was updated by the WHO in 2012 [9, 19]; however, the uptake of 3 doses was as low as $12 \%$ in 2014. Although the 3-dose recommendation was new in 2014 and despite it being a regional leader in IPTp uptake, Malawi still failed to meet its own 2011-2015 Malaria Strategic Plan target of having 80\% coverage of the uptake of 2 IPTp doses among pregnant women [18, 19, 21]. Nevertheless, increasing its uptake is crucial to reducing MIP burden. The effects of socio-demographics (e.g., education and parity) on IPTp uptake have been well documented [22-27]. For instance, a 2009 study conducted in 8 Malawian districts revealed that women who had secondary- or tertiary-level education were more likely to take the recommended number of IPTp doses than those without formal education [24]. However, studies have also reported contrasting findings regarding the influence of socio-demographics on IPTp uptake [22-25, 27]. Although a Tanzanian study found parity to be a crucial predictor of IPTp uptake [23], no association was reported in western Nigeria [28].

Although several studies have examined the effects of ANC attendance on IPTp uptake, the association has been inconsistent [26, 29-33]. For example, a Kenyan study found an association between the timing of first ANC visit and uptake of IPTp [29], whereas studies in Uganda, Tanzania, and Nigeria have reported no such association [34-37]. In Cameroon, less frequent clinic visits during pregnancy prevented the uptake of the recommended IPTp dose [38], whereas no such association was observed in Kenya [29]. The observed differences might be due to the various assessment durations; the Kenyan study [29] included not only currently pregnant women but also mothers with children aged under 1 year, whereas the Cameroonian study [38] was restricted to pregnant women. In addition, this inconsistency might be explained by the lack of consideration of the potential interactive effects of socio-demographics and ANC attendance on IPTp uptake.

Understanding the factors affecting IPTp uptake is critical for promoting and designing targeted interventions to improve the coverage of IPTp. Studies on the influence of both ANC attendance and socio-demographics on IPTp have focused only on their independent effects; thus far, limited empirical evidence is available on their interactive effects. Examining the effect modifiers of the relationship between ANC attendance and IPTp uptake is critical for identifying groups with a high risk of low IPTp uptake. Furthermore, although both the number of ANC visits and timing of first ANC visit are prominent factors, studies on ANC attendance and IPTp uptake have not elucidated how these two factors (i.e., number and timing) independently affect IPTp uptake, considering that the timing of first ANC visit may also influence the number of ANC visits, as reported in Tanzania [39].

Therefore, this study examined the relationship between ANC attendance, socio-demographics, and IPTp uptake among Malawian women by using a nationally representative sample. In particular, the study aimed to: (1) examine the factors (i.e., ANC attendance and socio-demographics) associated with the uptake of the recommended IPTp dose; (2) evaluate the independent association between the number of ANC visits and timing of first ANC visit on IPTp uptake; and, (3) investigate the moderating effects of socio-demographics on the relationship between ANC attendance and IPTp uptake.

\section{Methods}

\section{Study design and data source}

This cross-sectional study utilized data from the 2015 to 2016 Malawi Demographic and Health Survey (MDHS) [40]. The study design, methodology and sampling method have been described elsewhere in detail [40]. In summary, the sampling frame was the 2008 Malawi Population and Housing Census [40]. A two-stage stratified sampling method was used. The first stage involved the selection of standard enumeration areas (SEAs) from 
among 850 SEAs of Malawi. The second stage involved equal probability systematic selection of households in each SEA. Women aged 15-49 years who were either permanent residents of the selected households or visitors who stayed in the household the night before the survey were eligible to be interviewed. Data were collected from 9 October, 2015 to 17 February, 2016. The questionnaire was translated into Chichewa and Tumbuka (prominent local languages). Pre-testing was conducted in July and August 2015. All data collectors were trained and had experience with household surveys. Of 25,146 eligible women, 24,562 were successfully interviewed, indicating a $98 \%$ response rate.

To reduce recall bias and in line with the Roll Back Malaria Indicator on IPTp, the sample was restricted to women who had had a live birth in the 2 years preceding the survey [27, 41]. In total, 6549 women were finally included.

\section{Outcome variable}

As per WHO recommendations, the outcome measure was defined as the uptake of fewer than 3 IPTp doses (low uptake) or the uptake of 3 or more IPTp doses (recommended dose) [9].

\section{Independent variables}

ANC attendance, the main independent predictor for IPTp uptake in this study, was assessed using two items, namely 'number of ANC visits' and 'timing of first ANC visit' [25,38]. The number of ANC visits was categorized into two levels: adequate (4 or more) and inadequate (fewer than 4), according to WHO recommendations [9, 42]. The timing of first ANC visit was categorized according to the first, second, and third trimester.

Socio-demographics were also examined for their effects on IPTp uptake. In particular, three-level variables were used to categorize age (15-24, 25-34 and $\geq 35$ years), education (no formal education, primary education, secondary and above), marital status (married, never married, separated, widowed or divorced), and parity (primigravida, secundigravida, multigravida). Marital status was further assessed as a binary variable (married vs unmarried) in sub-group analysis. Principal component analysis was applied to the MDHS data for calculating wealth on the basis of household assets, such as bicycles and mobile phones. The calculated wealth was then categorized into quintiles, with the lowest to highest indicating the poorest to richest. In this study, wealth was categorized into a three-level variable by combining two sub-categories into single categories: poorest and poor into poor and rich and richest into rich, with the middle category unchanged.

\section{Statistical analyses}

The distribution of participants according to the number of ANC visits was analysed using the Chi square test. The distributions of ANC attendance and socio-demographics by IPTp uptake, along with their effects on the uptake, were further examined.

To investigate the association between the number of ANC visits and timing of first ANC visit on IPTp uptake, a single variable, ANC use, was generated and coded into the following 6 groups: (1) inadequate ANC visits and first ANC visit in first trimester; (2) inadequate ANC visits and first ANC visit in second trimester; (3) inadequate ANC visits and first ANC visit in third trimester; (4) adequate ANC visits and first ANC visit in first trimester; (5) adequate ANC visits and first ANC visit in second trimester; and, (6) adequate ANC visits and first ANC visit in third trimester. Group 3 (inferior situation) was used as the reference group, against which women from the other categories were compared. Logistic regression analyses were performed to estimate ORs and their 95\% CIs for the association between ANC use and recommended IPTp uptake.

Interactions of ANC attendance (i.e., number of ANC visits and timing of first ANC visit) and socio-demographics with IPTp uptake were examined. A $p$ of $<0.1$ was used for interaction terms to signal potential moderation [43], and a sub-group analysis was further used to examine the moderation effects.

All analyses considered the complex sample design, and only weighted data were used for analysis. Sample weighting allowed for adjustments to the cluster sampling design and sampling probabilities across clusters and strata. The level of significance was set at $p<0.05$ (two-tailed), and all analyses were performed using SPSS software version 22.0 (SPSS, Chicago, IL, USA).

\section{Ethical considerations}

Informed consent for the survey was obtained from each respondent at the start of each individual interview. Ethical approval was obtained from the Malawi National Health Sciences Research Committee of the Malawi Ministry of Health prior to the survey. The MDHS data sets are publicly available, and clearance to analyse them was provided by the International Classification of Functioning Disability and Health (ICF) under the Demographic Health Survey (DHS) programme.

\section{Results}

\section{Distribution of participants by number of ANC visits}

In total, 6549 women with a live birth in the 2 years prior to the survey participated in this study. Of them, $48.1 \%$ had adequate ANC visits. Table 1 displays the distribution of participants according to their number of ANC 
visits. The difference between women with adequate ANC visits and with inadequate ANC visits was significant $(p<0.05)$, in terms of age, marital status, education, occupation, wealth, residence, IPTp uptake, and timing of first ANC visit, but not parity and region.

\section{Distribution of participants by IPTp uptake and potential predictors recommended IPTp uptake}

Approximately $30.2 \%$ of the included women received the recommended IPTp dose. Distributions of potential predictors of IPTp uptake are listed in Table 2. Significant differences were observed between those with and without recommended IPTp uptake $(p<0.05)$, in terms of region, number of ANC visits, and timing of first ANC visit, but not the remaining variables. In the crude logistic regression analysis, age of $\leq 24$ years $(\mathrm{OR}=1.25,95 \%$ $\mathrm{CI}=1.03-1.51)$, adequate $\mathrm{ANC}$ visits $(\mathrm{OR}=1.68,95 \%$ $\mathrm{CI}=1.47-1.92)$, and first $\mathrm{ANC}$ visit in first $(\mathrm{OR}=2.72$, 95\% $\mathrm{CI}=1.97-3.76)$ and second $(\mathrm{OR}=2.30,95 \%$ $\mathrm{CI}=1.69-3.12)$ trimesters were significantly associated with increased odds of taking the recommended IPTp dose $(p<0.05)$.

\section{IPTp uptake stratified by number of ANC visits and timing of first ANC visit}

Table 3 presents the odds of recommended IPTp uptake stratified by the number of ANC visits and timing of first ANC visit. After adjustment for age, parity, marital status, education, wealth, and occupation, women who had reported for the first ANC visit in their first and second trimesters were more likely to take the recommended IPTp dose $(\mathrm{aOR}=1.97,95 \% \mathrm{CI}=1.40-2.76$ and $\mathrm{aOR}=1.93,95 \% \mathrm{CI}=1.42-2.64$, respectively) than women who reported in their third trimester. Similarly, women with adequate ANC visits were more likely to take the recommended dose than those with inadequate ANC visits $(\mathrm{aOR}=1.55,95 \% \mathrm{CI}=1.34-1.80)$.

Further examination of the association between the number of ANC visits and timing of first ANC visit (i.e., ANC use in the 6 groups) revealed that women with adequate ANC visits who had reported for their first ANC visit in the first or second trimester were approximately 3 times more likely to take the recommended IPTp dose $(\mathrm{aOR}=3.41,95 \% \mathrm{CI}=2.39-4.86$ and $\mathrm{aOR}=3.33,95 \%$ $\mathrm{CI}=2.36-4.70$, respectively), than women who had inadequate ANC visits and had reported for their first ANC visit in the third trimester. Moreover, women with adequate ANC visits who reported for their first ANC visit in the third trimester were approximately 5 times more likely to take the recommended IPTp dose $(\mathrm{aOR}=4.74$, $95 \% \mathrm{CI}=1.61-13.96)$ than women in the reference group.
Notably, among women with inadequate ANC visits, those who had reported for their first ANC visit in the first and second trimesters were approximately 2 times more likely to take the recommended dose $(\mathrm{OR}=2.24$, $95 \% \mathrm{CI}=1.44-3.49$ and $\mathrm{OR}=2.19,95 \% \mathrm{CI}=1.56-3.08$, respectively) than reference group women.

The design of the MDHS survey incorporates both residents of the selected households and visitors. Thus, in the sample, only 58 women $(0.9 \%)$ with a live birth 2 years before the survey were visitors. A sensitivity analysis to include only resident women for examination was further conducted and the results were consistent. Therefore, the findings from the whole sample were reported to comply with the MDHS survey design.

\section{Sub-group analysis for effects of socio-demographics on ANC attendance and recommended IPTp uptake}

The interaction terms (i.e., number of ANC visits and socio-demographics (i.e., residence, education, wealth, age, parity, and marital status)) on IPTp uptake $(p<0.1)$ were considered in sub-group analysis. In the subgroup analysis, the effect of the number of ANC visits on IPTp uptake was significant in the poor and middle wealth groups $(\mathrm{aOR}=1.94,95 \% \mathrm{CI}=1.39-2.71$ and $\mathrm{aOR}=1.75,95 \% \mathrm{CI}=1.45-2.11$, respectively), $25-34$ and $15-24$ years old women $(\mathrm{aOR}=2.02,95 \% \mathrm{CI}=1.59-2.06$ and $\mathrm{aOR}=1.48,95 \% \mathrm{CI}=1.19-1.83$, respectively), all levels of parity with higher odds for the multigravida group $(\mathrm{aOR}=1.74,95 \% \mathrm{CI}=1.42-2.13)$, and married women $(\mathrm{OR}=1.68,95 \% \mathrm{CI}=1.42-1.98$; Fig. 1). Furthermore, to examine ANC timing, reporting for the first ANC visit in the first and second trimesters (desirable ANC timing) was compared with reporting for the first ANC visit in the third trimester (undesirable ANC timing). The interaction between ANC timing and residence was significant $(p=0.014)$. The association between desirable ANC timing on IPTp uptake was significant only among rural residents $(\mathrm{aOR}=2.13,95 \% \mathrm{CI}=1.54-2.95$; Fig. 2).

\section{Discussion}

According to literature, this is the first study attempting to identify the association between timing of the first ANC visit and number of ANC visits, and also to evaluate the interactive effects of ANC attendance and socio-demographics on IPTp uptake. Having more ANC visits was expected to increase the likelihood of taking the recommended IPTp dose. However, the study revealed that although inadequate ANC visits $(<4)$ were noted, women who had their first ANC visit earlier in the first and second trimesters respectively had 2.24- and 2.19-fold increased odds of receiving the recommended IPTp dose. The effects of adequate ANC visits on taking the recommended IPTp dose were significant in married 
Table 1 Distribution of participants according to number of ANC visits

\begin{tabular}{|c|c|c|c|c|}
\hline \multirow[t]{3}{*}{ Variable } & \multirow{3}{*}{$\begin{array}{l}\text { Total }(n=6549) \\
n\end{array}$} & \multicolumn{2}{|c|}{ Number of ANC visit categories } & \multirow[t]{3}{*}{$p$ value $^{b}$} \\
\hline & & Inadequate $^{a}(n=3402)$ & Adequate $^{\mathrm{a}}(n=3147)$ & \\
\hline & & $n(\%)$ & $n(\%)$ & \\
\hline Age (years) & & & & $0.035^{*}$ \\
\hline $15-24$ & 3043 & $1642(48.3)$ & $1401(44.5)$ & \\
\hline $25-34$ & 2517 & $1246(36.6)$ & $1271(40.4)$ & \\
\hline$\geq 35$ & 989 & $514(15.1)$ & $475(15.1)$ & \\
\hline Marital status & & & & $0.012^{*}$ \\
\hline Married & 5470 & $2799(82.3)$ & $2671(84.9)$ & \\
\hline Never married & 346 & $210(6.2)$ & $136(4.3)$ & \\
\hline Separated/divorced/widowed & 733 & $393(11.6)$ & $340(10.8)$ & \\
\hline Education & & & & $<0.001^{* * *}$ \\
\hline No formal education & 785 & $444(13.0)$ & $341(10.8)$ & \\
\hline Primary & 4382 & $2350(69.1)$ & $2032(64.6)$ & \\
\hline Secondary and above & 1382 & $608(17.9)$ & $774(24.6)$ & \\
\hline Occupation & & & & $0.049^{*}$ \\
\hline Unemployed & 2086 & $1130(33.2)$ & $956(30.4)$ & \\
\hline Employed & 4463 & $2272(66.8)$ & $2191(69.6)$ & \\
\hline Parity & & & & 0.132 \\
\hline Primigravida & 1818 & $899(26.4)$ & $919(29.2)$ & \\
\hline Secundigravida & 1352 & $722(21.2)$ & $630(20.0)$ & \\
\hline Multigravida & 3379 & $1781(52.4)$ & $1598(50.8)$ & \\
\hline Wealth index & & & & $<0.001^{* * *}$ \\
\hline Poor & 3147 & $1724(50.7)$ & $1423(45.2)$ & \\
\hline Middle & 1261 & $690(20.3)$ & $571(18.2)$ & \\
\hline Rich & 2141 & $988(29.0)$ & $1153(36.6)$ & \\
\hline Residence & & & & $<0.001^{* * *}$ \\
\hline Urban & 897 & $381(11.2)$ & $516(16.4)$ & \\
\hline Rural & 5652 & $3021(88.8)$ & $2631(83.6)$ & \\
\hline Region & & & & 0.841 \\
\hline Northern & 748 & $389(11.4)$ & $359(11.4)$ & \\
\hline Central & 2756 & $1418(41.7)$ & $1338(42.5)$ & \\
\hline Southern & 3045 & $1595(46.9)$ & $1450(46.1)$ & \\
\hline IPTp uptake & & & & $<0.001^{* * *}$ \\
\hline$<3$ & 4568 & $2551(75.0)$ & $2017(64.1)$ & \\
\hline$\geq 3$ & 1981 & $850(25.0)$ & $1131(35.9)$ & \\
\hline Timing of first ANC visit $(n=6413)$ & & & & $<0.001^{* * *}$ \\
\hline First trimester & 1503 & 325 (9.9) & 1178 (37.5) & \\
\hline Second trimester & 4427 & $2492(76.2)$ & 1935 (61.6) & \\
\hline Third trimester & 483 & $452(13.8)$ & $31(1.0)$ & \\
\hline
\end{tabular}

ANC antenatal care, IPTp intermittent preventive treatment for malaria during pregnancy

${ }^{*} p<0.05 ; * * 0<0.01 ; * * * 0.001$

a The number of ANC visits was categorized into two levels: adequate ANC visits (4 or more) and inadequate ANC visits (fewer than 4)

${ }^{b} p$ value of Pearson's Chi squared test

women, those aged $\leq 34$ years, and those in the middle or poor wealth categories, whereas early ANC initiation was associated with an increased likelihood of taking the recommended IPTp dose among rural women only.
The rate of recommended IPTp uptake was $30.2 \%$. A substantially lower rate (11\%) was reported in Simuyu, Tanzania [44]. The change in the WHO IPTp policy in 2014 (minimum number of doses from 2 to 3 ) may have 
Table 2 Distribution of participants by uptake of IPTp and potential factors affecting uptake

\begin{tabular}{|c|c|c|c|c|c|}
\hline Variable & $\begin{array}{l}<3 \text { doses }(n=4568) \\
n(\%)\end{array}$ & $\begin{array}{l}\geq 3 \text { doses }(n=1981) \\
n(\%)\end{array}$ & $p$ value $^{a}$ & OR & $(95 \% \mathrm{Cl})$ \\
\hline Age (years) & & & 0.055 & & \\
\hline $15-24$ & $2070(45.3)$ & $973(49.1)$ & & 1.25 & $1.03-1.51^{*}$ \\
\hline $25-34$ & $1780(39.0)$ & $737(37.2)$ & & 1.09 & $0.89-1.35$ \\
\hline$\geq 35$ & $718(15.7)$ & $271(13.7)$ & & 1.00 & \\
\hline Marital status & & & 0.505 & & \\
\hline Never married & $253(5.5)$ & $92(4.7)$ & & 0.84 & $0.62-1.13$ \\
\hline Separated/divorced/widowed & $509(11.1)$ & $225(11.3)$ & & 1.01 & $0.82-1.25$ \\
\hline Married & $3806(83.3)$ & $1664(84.0)$ & & 1.00 & \\
\hline Education & & & 0.790 & & \\
\hline No formal education & $538(11.8)$ & $247(12.5)$ & & 1.06 & $0.82-1.35$ \\
\hline Primary & $3067(67.1)$ & $1314(66.3)$ & & 0.98 & $0.82-1.18$ \\
\hline Secondary and above & $963(21.1)$ & $420(21.2)$ & & 1.00 & \\
\hline Occupation & & & 0.313 & & \\
\hline Employed & $3135(68.6)$ & $1329(67.1)$ & & 0.93 & $0.81-1.07$ \\
\hline Unemployed & $1433(31.4)$ & $652(32.9)$ & & 1.00 & \\
\hline Parity & & & 0.373 & & \\
\hline Primigravida & $1242(27.2)$ & $575(29.0)$ & & 1.12 & $0.95-1.32$ \\
\hline Secundigravida & $936(20.5)$ & $417(21.0)$ & & 1.08 & $0.89-1.29$ \\
\hline Multigravida & $2390(52.3)$ & $989(49.9)$ & & 1.00 & \\
\hline Wealth index & & & 0.886 & & \\
\hline Rich & $1484(32.5)$ & $656(33.1)$ & & 0.98 & $0.84-1.15$ \\
\hline Middle & $888(19.4)$ & $374(18.9)$ & & 0.95 & $0.79-1.16$ \\
\hline Poor & $2196(48.0)$ & $951(48.0)$ & & 1.00 & \\
\hline Residence & & & 0.251 & & \\
\hline Urban & $649(14.2)$ & $248(12.5)$ & & 0.87 & $0.68-1.11$ \\
\hline Rural & $3919(85.8)$ & $1733(87.5)$ & & 1.00 & \\
\hline Region & & & $0.044^{*}$ & & \\
\hline Northern & $550(12.0)$ & $198(10.0)$ & & 0.86 & $0.70-1.06$ \\
\hline Central & $1872(41.0)$ & $884(44.6)$ & & 1.13 & $0.97-1.32$ \\
\hline Southern & $2146(47.0)$ & $899(45.4)$ & & 1.00 & \\
\hline ANC visits & & & $<0.001^{* * *}$ & & \\
\hline Adequate $(\geq 4)$ & $2017(44.2)$ & $1131(57.1)$ & & 1.68 & $1.47-1.92^{* * *}$ \\
\hline Inadequate $(<4)$ & $2551(55.8)$ & $850(42.9)$ & & 1.00 & \\
\hline First ANC visit timing $(n=6413)$ & & & $<0.001^{* * *}$ & & \\
\hline First trimester & $985(22.1)$ & $518(26.4)$ & & 2.72 & $1.97-3.76^{* * *}$ \\
\hline Second trimester & 3065 (68.8) & 1362 (69.6) & & 2.30 & $1.69-3.12^{* * *}$ \\
\hline Third trimester & 405 (9.1) & $78(4.0)$ & & 1.00 & \\
\hline
\end{tabular}

ANC antenatal care, IPTp intermittent preventive treatment for malaria during pregnancy, $\mathrm{Cl}$ confidence interval, $\mathrm{OR}$ odds ratio

${ }^{*} p<0.05 ;{ }^{* *} p<0.01 ;{ }^{* * *} p<0.001$

a $p$ value of Pearson's Chi squared test

been partially responsible for this low uptake because most countries were still in the process of adopting the new guidelines. In the current study, only $48.1 \%$ of women had adequate ANC visits, similar to the $46 \%$ reported in an earlier Malawian study [45]. These results suggest that IPTp coverage and ANC attendance remain a challenge. The results increase the understanding of the factors affecting the uptake of such services, particularly emphasizing the associated effects of ANC attendance; further relevant research is warranted.

This study determined that women with adequate ANC visits were more likely to take the recommended IPTp dose, consistent with studies conducted in Cameroon and Ghana [31,33]. This may be because IPTp 
Table 3 Association between the number of ANC visits and IPTp uptake stratified by ANC timing

\begin{tabular}{|c|c|c|c|}
\hline \multirow[t]{2}{*}{ Timing of the first ANC visit } & \multicolumn{2}{|c|}{ Number of ANC visit categories $\mathrm{OR}^{\mathrm{a}}(95 \% \mathrm{Cl})$} & \multirow[t]{2}{*}{ Total } \\
\hline & Inadequate ${ }^{b}$ & Adequate $^{\mathbf{b}}$ & \\
\hline First trimester & $2.24(1.44-3.49 * * *)$ & $3.41\left(2.39-4.86^{* * *}\right)$ & $1.97\left(1.40-2.76^{* * *}\right)$ \\
\hline Second trimester & $2.19\left(1.56-3.08^{* * *}\right)$ & $3.33\left(2.36-4.70^{* * *}\right)$ & $1.93\left(1.42-2.64^{* * *}\right)$ \\
\hline Third trimester & 1.00 & $4.74\left(1.61-13.96^{* *}\right)$ & 1.00 \\
\hline Total & 1.00 & $1.55\left(1.34-1.80^{* * *}\right)$ & \\
\hline
\end{tabular}

ANC antenatal care, IPTp intermittent preventive treatment for malaria during pregnancy, $\mathrm{Cl}$ confidence interval, OR odds ratio

${ }^{*} p<0.05$; ${ }^{* *} p<0.01$; *** $p<0.001$

a Adjusted for age, parity, marital status, education, wealth, and occupation

b The number of ANC visits was categorized into two levels: adequate ANC visits (four or more) and inadequate ANC visits (fewer than four)

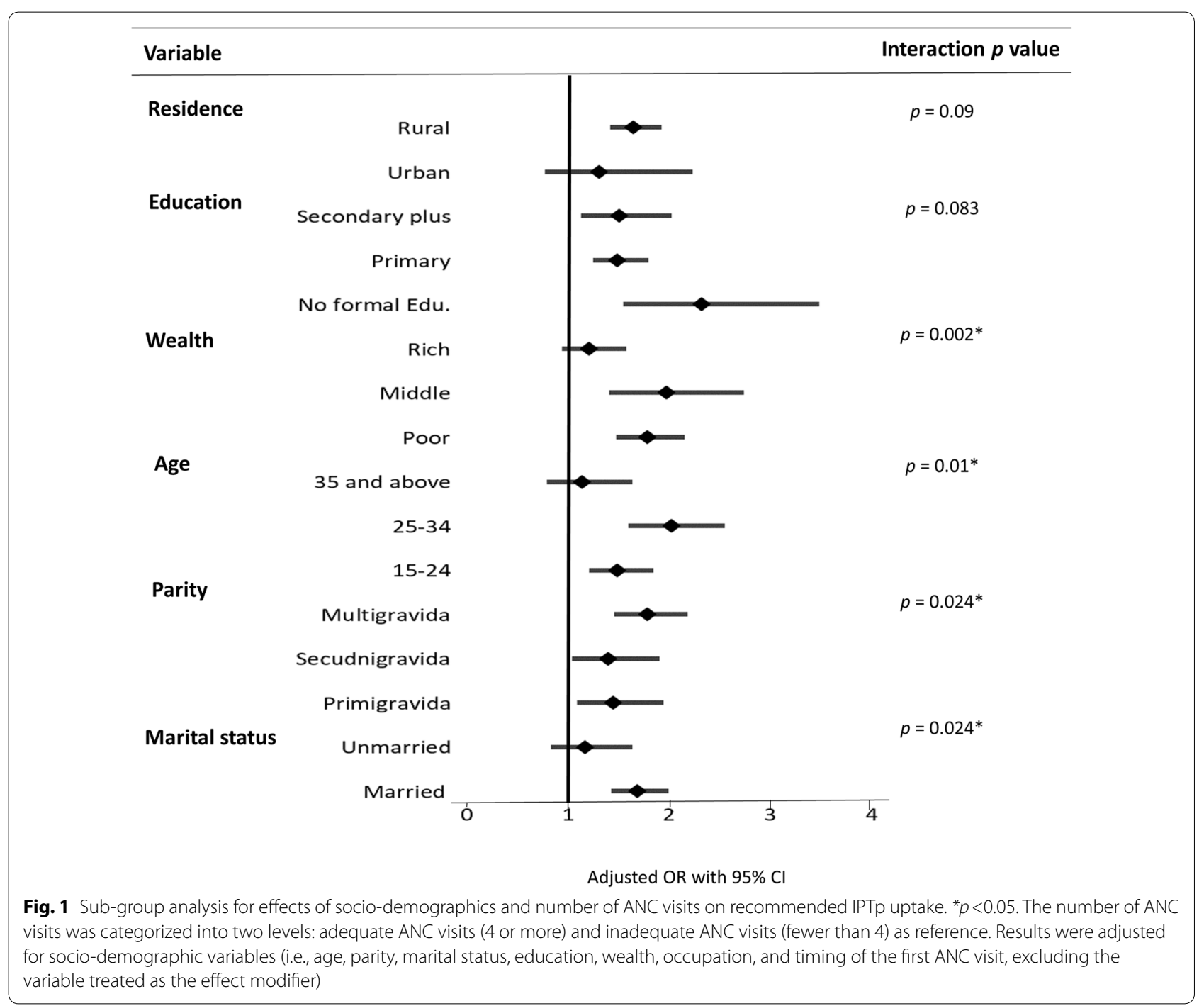

policies in these countries recommend that IPTp should be administered at ANC visits, preferably as direct observation therapy (DOT). Consequently, women with higher numbers of ANC visits are more likely to complete the required IPTp doses. By contrast, the Kenyan study found no such association [29]. Other factors, such as availability of drugs at ANC clinics and effective DOT implementation, might also contribute 


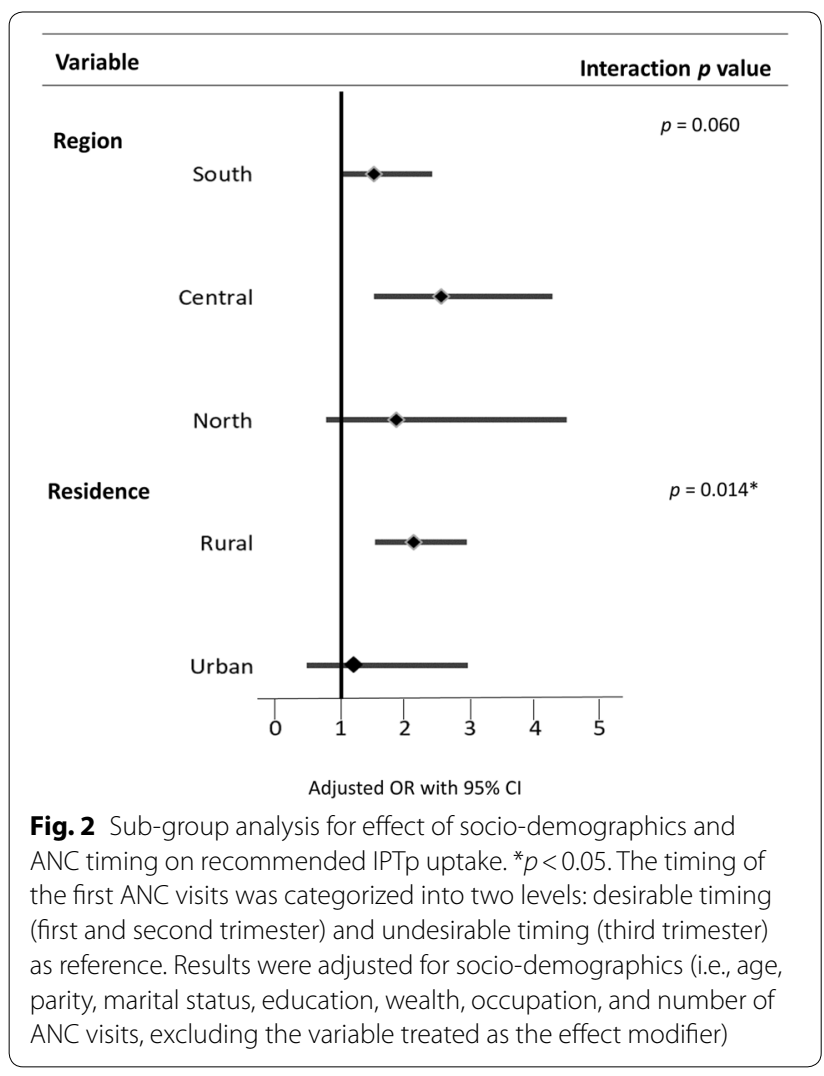

to the observed differences [37, 46]. Additional studies are required to understand the health facility-based factors that could influence IPTp uptake, such as accessibility of drugs at clinics and availability of clean water for DOT.

The current study's results support the findings observed in Tanzania and Ghana [23, 33], where women with ANC initiation in the first and second trimesters reported a higher IPTp uptake. However, other authors [34-37, 47] have reported no such association, potentially because of the different designs employed in these studies; for instance, Kibusi et al. [23] conducted a quantitative study, whereas Rass et al. [37] conducted a qualitative study. If a woman reports for ANC in the third trimester, she may not receive the full IPTp dose because of the delay in initiation of IPTp uptake.

Compared with other studies [23, 25, 26, 33, 38], this study further demonstrated the independent association between timing of the first ANC visit, in addition to the number of ANC visits, and IPTp uptake among pregnant women. In particular, the results suggest that reporting for the first ANC earlier during pregnancy, despite having an inadequate number of ANC visits, was associated with recommended IPTp uptake. ANC clinics are the main sources of disseminating crucial health information to women during pregnancy. Therefore, women may be advised on the importance of IPTp when they visit the ANC clinic early, and therefore, they may be able to source the drug from elsewhere if they fail to attend subsequent $\mathrm{ANC}$ visits [48].

Increased odds of recommended IPTp uptake in those with adequate visits despite having late initiation of ANC were also observed, but with a wider confidence interval, possibly because of the smaller sample number in this category. Two Ghanaian studies reported that 4 or more ANC visits were associated with higher IPTp uptake [26, 33]. The current findings further revealed the significance of this factor, independent of the timing of the first ANC visit, suggesting the need for healthcare workers to encourage late ANC initiators to make frequent ANC visits. However, women who report late for ANC with complications, such as malaria infection, may make frequent clinic visits and subsequently receive more SP as remedial treatment. In addition, the observed increased odds should be interpreted with caution because women who reported for ANC early in the third trimester may have completed the recommended IPTp dose, but those who reported late in the third trimester may not have.

Studies have reported age and marital status [23, 49], wealth $[24,30]$, education $[23,24,30]$, and parity $[23,26$, 30] to be significant predictors for IPTp uptake. However, this study did not observe a significant association between socio-demographics and IPTp uptake. Instead, moderating effects of socio-demographics on the association between ANC attendance and IPTp uptake were found in the sub-group analyses. Specifically, the effect of adequate ANC visits on taking the recommended IPTp dose was significant among married women, those in the poor and middle wealth groups, and women aged $\leq 34$ years $(p<0.05)$. Notably, this relationship was significant at all levels of parity, with the highest odds observed for the multigravida group. In addition, the effect of desirable ANC timing on IPTp uptake was significant only among rural residents. Thus, tailor-made behavioural-change messages on IPTp uptake for different vulnerable groups of women are required to ensure effective scale-up of IPTp uptake.

The use of a nationally representative sample could strengthen the generalization of these results to Malawian women; however, because information on IPTp uptake and ANC attendance relied on recall, recall bias may have occurred. Recall ability is considered to be associated with the vividness, meaningfulness and significance of the event [50]. Recall may be better for less frequent and more significant events, such as the ones assessed in this study (IPTp uptake and ANC attendance), however, recall bias might still have compromised the findings. The MDHS data used for the analysis have not been verified with participants' recalled information 
for either IPTp uptake or ANC attendance from the mothers on the ANC cards, which might have helped in reducing recall bias. Future studies should consider verifying recalled information by using the mothers' ANC or health cards. Limiting the participants to those with a live birth in the 2 years preceding the survey also may have helped to reduce recall bias. Crucial factors, such as previous malaria infection, infection of family members, and health facility-based factors (i.e., drug stock-outs and ANC health worker attitude), which might affect pregnant mothers' intention to return to ANC and thus their IPTp uptake, could not be evaluated in this study. Finally, because this was a cross-sectional study, causality could not be inferred.

\section{Conclusion}

ANC attendance, namely the number of ANC visits and timing of the first visit, strongly influences IPTp uptake among Malawian women. The results demonstrated the independent association of these two ANC attendance assessments on IPTp uptake. In addition to frequent ANC visits, earlier ANC initiation should also be emphasized, which may simultaneously facilitate recommended IPTp uptake to appropriately protect pregnant women from the risk of malaria infection. The significant moderating effects of socio-demographics can facilitate the identification and targeting of vulnerable groups to promote the recommended IPTp uptake rate in Malawi. Further research is required to examine the effects of health facility-based factors on IPTp use and the effects of IPTp dose on birth outcomes. Qualitative studies interviewing both women and health personnel are also required for further elucidating barriers to recommended IPTp uptake.

\section{Abbreviations \\ IPTp: intermittent preventive treatment for malaria during pregnancy; MIP: malaria in pregnancy; ANC: antenatal care; MoH: Ministry of Health; WHO: World Health Organization; aOR: adjusted odds ratio; SP: sulfadoxine- pyrimethamine; DOT: direct observed therapy; DHS: demographic health surveys; MDHS: Malawi Demgraphic Health Survey; SEAs: standard enumera- tion areas; SPSS: Statistical Packages for Social Sciences; ICF: International Classification of Functioning Disability and Health.}

\section{Authors' contributions}

ON is a Malawian scientist, who worked on Maternal and Child Health programmes in Malawi, including a malaria programme with World Vision, Malawi, from 2015 to 2016; he is currently enrolled in a Master of Science programme in the School of Public Health, Taipei Medical University. ON conducted the data analysis, interpreted the data and drafted the manuscript. TWC assisted in the literature review and provided suggestions for manuscript preparation. YHC conceived and designed this study and supervised all critical data analysis and manuscript preparation. All authors read and approved the final manuscript.

\section{Author details}

${ }^{1}$ School of Public Health, College of Public Health, Taipei Medical University, Taipei, Taiwan. ${ }^{2}$ Department of Molecular Parasitology and Tropical Diseases, School of Medicine, College of Medicine, Taipei Medical University, Taipei, Taiwan.

\section{Acknowledgements}

We acknowledge the International Classification of Functioning Disability and Health (ICF) for the permission to use the MDHS data set for analysis.

\section{Competing interests}

The authors declare that they have no competing interests.

\section{Availability of data and materials}

The study used, with permission, data from the International Classification of Functioning, Disability, and Health (ICF). The data are publicly available and may be requested from the ICF on (https://dhsprogram.com/data/avail able-datasets.cfm). The questionnaire used for the analyses is the women's questionnaire contained within the MDHS, which can be accessed publicly at https://dhsprogram.com/pubs/pdf/FR319/FR319.pdf.

\section{Consent for publication \\ Not applicable.}

\section{Ethics approval and consent to participate}

Approval for data collection from the DHS was provided by the Malawi National Health Sciences Research Committee of the Malawi MoH. All interviewed participants provided informed consent to participate in the 2015-2016 MDHS and for the information from the survey to be published. The data used in this analysis were anonymous, with no individual names of participants captured.

\section{Funding}

None.

\section{Publisher's Note}

Springer Nature remains neutral with regard to jurisdictional claims in published maps and institutional affiliations.

Received: 15 November 2017 Accepted: 16 May 2018

Published online: 25 May 2018

References

1. WHO. World malaria report 2017. Geneva: World Health Organization; 2017.

2. Guyatt HL, Snow RW. The epidemiology and burden of Plasmodium falciparum-related anemia among pregnant women in sub-Saharan Africa. Am J Trop Med Hyg. 2001;64(suppl 1):36-44.

3. Desai M, Ter Kuile FO, Nosten F, McGready R, Asamoa K, Brabin B, et al. Epidemiology and burden of malaria in pregnancy. Lancet Infect Dis. 2007;7:93-104.

4. Lawn JE, Blencowe H, Waiswa P, Amouzou A, Mathers C, Hogan D, et al. Stillbirths: rates, risk factors, and acceleration towards 2030. Lancet. 2016;387:587-603.

5. Boudová S, Divala T, Mawindo P, Cohee L, Kalilani-Phiri L, Thesing P, et al. The prevalence of malaria at first antenatal visit in Blantyre, Malawi declined following a universal bed net campaign. Malar J. 2015;14:422.

6. Hennessee I, Chinkhumba J, Briggs-Hagen M, Bauleni A, Shah MP, Chalira A, et al. Household costs among patients hospitalized with malaria: evidence from a national survey in Malawi, 2012. Malar J. 2017;16:395.

7. USAID. President's Malaria Initiative; Malawi Operational Plan FY17. 2017. https://www.pmi.gov/docs/default-source/default-document-library/ malaria-operational-plans/fy17/fy-2017-malawi-malaria-operational-plan. pdf?sfvrsn=6. Accessed 10 Mar 2018.

8. WHO. Intermittent preventive treatment in pregnancy (IPTp). Geneva: World Health Organization; 2017. http://www.who.int/malaria/areas/ preventive_therapies/pregnancy/en/. Accessed 10 Mar 2018. 
9. WHO. intermittent preventive treatment of malaria in pregnancy using sulfadoxine pyrimethamine (IPTp-SP): updated WHO policy recommendation (October 2012). Geneva: World Health Organization; 2012. http://www.who.int/malaria/iptp_sp_updated_policy_recommenda tion_en_102012.pdf?ua=1. Accessed 24 Mar 2018.

10. Bhatt S, Weiss DJ, Cameron E, Bisanzio D, Mappin B, Dalrymple U, et al. The effect of malaria control on Plasmodium falciparum in Africa between 2000 and 2015. Nature. 2015;526:207.

11. Rogerson SJ, Chaluluka E, Kanjala M, Mkundika P, Mhango C, Molyneux ME. Intermittent sulfadoxine-pyrimethamine in pregnancy: effectiveness against malaria morbidity in Blantyre, Malawi, in 1997-1999. Trans R Soc Trop Med Hyg. 2000;94:549-53.

12. WHO. Intermittent preventive treatment of malaria in pregnancy (IPTp) with sulfadoxine-pyrimethamine (SP). Geneva: World Health Organization; 2012. http://www.who.int/malaria/mpac/sep2012/iptp_sp_erg_ meeting_report_july2012.pdf. Accessed 10 Mar 2018.

13. Feng G, Simpson JA, Chaluluka E, Molyneux ME, Rogerson SJ. Decreasing burden of malaria in pregnancy in Malawian women and its relationship to use of intermittent preventive therapy or bed nets. PLOS ONE. 2010;5:e12012.

14. Wallon M, Agarwal S, Roman E, Dickerson A. A malaria in pregnancy country case study: Malawis successes and remaining challenges for malaria in pregnancy programming. https://www.mchip.net/sites/defau It/files/Malawi\%20MIP\%20Case\%20Study_final.pdf. Accessed 10 Mar 2018.

15. WHO. Technical expert group meeting on intermittent preventive treatment in pregnancy (IPTp), Geneva: World Health Organization; 11-13 July 2007. http://apps.who.int/iris/bitstream/10665/43892/1/9789241596 640_eng.pdf. Accessed 10 Mar 2018.

16. Agarwal K, Alonso P, Chico RM, Coleman J, Dellicour S, Hill J, et al. Global call to action to scale-up coverage of intermittent preventive treatment of malaria in pregnancy: seminar report. Malar J. 2015;14:206.

17. Chico RM, Dellicour S, Roman E, Mangiaterra V, Coleman J, Menendez C, et al. Global call to action: maximize the public health impact of intermittent preventive treatment of malaria in pregnancy in sub-Saharan Africa. Malar J. 2015;14:207.

18. Bausell $L$, Wolf K. Treatment uptake and availability of antimalarial drugs for intermittent preventative treatment in pregnant women in Malawi. USAID, Deliver Project, Task Order 7; 2015. http://apps.who.int/medic inedocs/documents/s22060en/s22060en.pdf. Accessed 10 Mar 2018.

19. National Malaria Control Programme - NMCP/Malawi and ICF International, Malawi Malaria Indicator Survey. 2015, NMCP/Malawi and ICF international: Rockville. USA: Maryland; 2014.

20. van Eijk AM, Hill J, Alegana VA, Kirui V, Gething PW, ter Kuile FO, et al. Coverage of malaria protection in pregnant women in sub-Saharan Africa: a synthesis and analysis of national survey data. Lancet Infect Dis. 2011;11:190-207.

21. Malawi Ministry of Health. Malaria strategic plan 2011-2015 towards universal access. National Malaria Control Programme Community Health Sciences Unit. https://www.medbox.org/malaria-strategicplan-2011-2015/download.pdf. Accessed 10 Mar 2018.

22. Fokam EB, Ngimuh L, Anchang-Kimbi JK, Wanji S. Assessment of the usage and effectiveness of intermittent preventive treatment and insecticide-treated nets on the indicators of malaria among pregnant women attending antenatal care in the Buea Health District, Cameroon. Malar J. 2016;15:172.

23. Kibusi SM, Kimunai E, Hines CS. Predictors for uptake of intermittent preventive treatment of malaria in pregnancy (IPTp) in Tanzania. BMC Public Health. 2015;15:540.

24. Mwandama D, Gutman J, Wolkon A, Luka M, Jafali J, Ali D, et al. The use of intermittent preventive treatment in pregnancy and insecticide-treated bed nets for malaria prevention by women of child-bearing age in eight districts in Malawi. Malar J. 2015;14:316.

25. Protas J, Tarimo D, Moshiro C. Determinants of timely uptake of ITN and SP (IPT) and pregnancy time protected against malaria in Bukoba, Tanzania. BMC Res Notes. 2016:9:318.

26. Stephen AA, Wurapa F, Afari EA, Sackey SO, Malm KL, Nyarko KM. Factors influencing utilization of intermittent preventive treatment for pregnancy in the Gushegu district, Ghana, 2013. Pan Afr Med J. 2016;25(Suppl 1):4.
27. Wanzira H, Katamba H, Okullo AE, Rubahika D. The challenge of using intermittent preventive therapy with sulfadoxine/pyrimethamine among pregnant women in Uganda. Malar J. 2016;15:401.

28. Amoran OE, Ariba AA, Iyaniwura CA. Determinants of intermittent preventive treatment of malaria during pregnancy (IPTp) utilization in a rural town in Western Nigeria. Reprod Health. 2012;9:12.

29. Hill J, Dellicour S, Bruce J, Ouma P, Smedley J, Otieno P, et al. Effectiveness of antenatal clinics to deliver intermittent preventive treatment and insecticide treated nets for the control of malaria in pregnancy in Kenya. PLOS ONE. 2013;8:e64913.

30. Hill J, Hoyt J, van Eijk AM, D'Mello-Guyett L, ter Kuile FO, Steketee R, et al. Factors affecting the delivery, access, and use of interventions to prevent malaria in pregnancy in sub-Saharan Africa: a systematic review and meta-analysis. PLoS Med. 2013;10:e1001488.

31. Leonard N, Eric FB, Judith AK, Samuel W. Factors associated to the use of insecticide treated nets and intermittent preventive treatment for malaria control during pregnancy in Cameroon. Arch Public Health. 2016;74:5.

32. Ndyomugyenyi R, Katamanywa J. Intermittent preventive treatment of malaria in pregnancy (IPTp): do frequent antenatal care visits ensure access and compliance to IPTp in Ugandan rural communities? Trans $R$ Soc Trop Med Hyg. 2010;104:536-40.

33. Owusu-Boateng I, Anto F. Intermittent preventive treatment of malaria in pregnancy: a cross-sectional survey to assess uptake of the new sulfadoxine-pyrimethamine five dose policy in Ghana. Malar J. 2017;16:323.

34. Anders K, Marchant T, Chambo P, Mapunda P, Reyburn H. Timing of intermittent preventive treatment for malaria during pregnancy and the implications of current policy on early uptake in north-east Tanzania. Malar J. 2008;7:79.

35. Gross K, Alba S, Schellenberg J, Kessy F, Mayumana I, Obrist B. The combined effect of determinants on coverage of intermittent preventive treatment of malaria during pregnancy in the Kilombero Valley, Tanzania. Malar J. 2011;10:140.

36. Onoka CA, Hanson K, Onwujekwe OE. Low coverage of intermittent preventive treatment for malaria in pregnancy in Nigeria: demand-side influences. Malar J. 2012;11:82.

37. Rassi C, Graham K, Mufubenga P, King R, Meier J, Gudoi SS. Assessing supply-side barriers to uptake of intermittent preventive treatment for malaria in pregnancy: a qualitative study and document and record review in two regions of Uganda. Malar J. 2016;15:341.

38. Anchang-Kimbi JK, Achidi EA, Apinjoh TO, Mugri RN, Chi HF, Tata RB, et al. Antenatal care visit attendance, intermittent preventive treatment during pregnancy (IPTp) and malaria parasitaemia at delivery. Malar J. 2014;13:162.

39. Gupta S, Yamada G, Mpembeni R, Frumence G, Callaghan-Koru JA, Stevenson $\mathrm{R}$, et al. Factors associated with four or more antenatal care visits and its decline among pregnant women in Tanzania between 1999 and 2010. PLoS ONE. 2014;9:e101893.

40. National Statistical Office (NSO) [Malawi] and ICF 2017. Malawi demographic and health survey 2015-2016. Zomba: NSO and ICF; 2017.

41. Roll Back Malaria, MEASURE Evaluation, USAID, UNICEF, World Health Organization, MACEPA, CDC. Guidelines for core population-based indicators. Calverton: MEASURE evaluation. https://reliefweb.int/sites/reliefweb. int/files/resources/AC719D00E5DE6F5D492575B3001BE5A3-RMB-quide line-20009.pdf. Accessed 10 Mar 2018.

42. WHO. Recommendations on antenatal care for a positive pregnancy experience. Geneva: World Health Organization; 2016. http://apps.who. int/iris/bitstream/10665/250796/1/9789241549912-eng.pdf. Accessed 10 Mar 2018.

43. Evans EA, Upchurch DM, Simpson T, Hamilton AB, Hoggatt KJ. Differences by veteran/civilian status and gender in associations between childhood adversity and alcohol and drug use disorders. Soc Psychiatry Psychiatr Epidemiol. 2018:53:421-35.

44. Sambili B, Kimambo R, Peng Y, Ishunga E, Matasha E, Matumu G, et al. Factors influencing anti-malarial prophylaxis and iron supplementation non-compliance among pregnant women in Simiyu Region, Tanzania. Int J Environ Res Public Health. 2016;13:e626.

45. Roberts J, Sealy D, Marshak HH, Manda-Taylor L, Gleason P, Mataya R. The patient-provider relationship and antenatal care uptake at two referral hospitals in Malawi: a qualitative study. Malawi Med J. 2015;27:145-50.

46. Ameh S, Owoaje E, Oyo-Ita A, Kabiru CW, Akpet OE, Etokidem A, et al. Barriers to and determinants of the use of intermittent preventive treatment 
of malaria in pregnancy in Cross River State, Nigeria: a cross-sectional study. BMC Pregnancy Childbirth. 2016;16:99.

47. Doku DT, Zankawah MM, Adu-Gyamfi AB. Factors influencing dropout rate of intermittent preventive treatment of malaria during pregnancy. BMC Res Notes. 2016;9:460.

48. Sangaré LR, Stergachis A, Brentlinger PE, Richardson BA, Staedke SG, Kiwuwa MS, et al. Determinants of use of intermittent preventive treatment of malaria in pregnancy: Jinja, Uganda. PLoS One. 2010;5:e15066.

49. Choonara S, Odimegwu CO, Elwange BC. Factors influencing the usage of different types of malaria prevention methods during pregnancy in Kenya. Afr Health Sci. 2015;15:413-9.

50. Coughlin SS. Recall bias in epidemiologic studies. J Clin Epidemiol. 1990;43:87-91.
Ready to submit your research? Choose BMC and benefit from:

- fast, convenient online submission

- thorough peer review by experienced researchers in your field

- rapid publication on acceptance

- support for research data, including large and complex data types

- gold Open Access which fosters wider collaboration and increased citations

- maximum visibility for your research: over $100 \mathrm{M}$ website views per year

At BMC, research is always in progress.

Learn more biomedcentral.com/submissions 